\title{
EFICACIA Y EFICIENCIA DE LEADER II EN ANDALUCÍA: APROXIMACIÓN A UN ÍNDICE-RESULTADO EN MATERIA DE TURISMO RURAL ${ }^{1, *}$
}

\author{
D. Márquez Fernández ${ }^{(1)}, C$. Foronda Robles(2), \\ L. Galindo Pérez de Azpillaga ${ }^{(3)}$ y A. García López ${ }^{(4)}$ \\ Grupo de investigación "Estudios Integrados de Geografía" (HUM- 601). \\ Universidad de Sevilla. C/ María de Padilla s/n, 41004 Sevilla. \\ (1) marquezd@us.es (2) foronda@us.es (3) Igalindo@us.es (4) angar@us.es
}

\begin{abstract}
Resumen: La evaluación se ha convertido en un elemento estratégico de diferenciación, en la medida en que permite demostrar a sus propios beneficiarios, que la aplicación de los recursos públicos o privados, es la más adecuada posible. Para evaluar los programas de desarrollo rural es necesario un sistema de indicadores, que responden a cinco grandes tipos de los que nos centraremos en dos: eficiencia (el esfuerzo invertido en términos de recursos físicos, humanos o financieros y los resultados obtenidos) y eficacia (los impactos o resultados del programa respecto a sus objetivos). En este articulo tratamos de poner de manifiesto como la evaluación de la medida B.3 (actividades turísticas) permite, a través del análisis de la eficacia (proyectos) y la eficiencia (inversión) en LEADER II, una primera aproximación en la construcción de un índice resultado.
\end{abstract}

Palabras clave: Eficacia, eficiencia, LEADER II, turismo rural, sostenibilidad,

\begin{abstract}
The evaluation has turned into a strategic element of differentiation, into the measurement into that allows to demonstrate his own beneficiaries, that the application of the public or private resources, it is as suitable as possible. To evaluate is necessary a system of indicators, which answer to five big types of which we will centre on two: efficiency (the effort inverted in terms of physical, human or financial resources and the obtained results) and effectiveness (the impacts or results of the program with regard to his aims). In this article we try to put of manifest as the evaluation of the measurement B.3 (tourist activities) it allows across the analysis of the efficiency (projects) and the effectiveness(inves(ment) in LEADER II, one approximation in the construction of a proved index.
\end{abstract}

Key words: Efficiency, effectiveness, LEADER II, rural tourism, rural sustainability.

* Recibido: 04-04-05. Aceptado: 23-06-05. 


\section{Introducción}

Este artículo se enmarca en el proyecto "Construcción de una red de indicadores de sostenibilidad para la evaluación dinámica de los procesos de desarrollo rural áreas LEADER y PRODER". Se trata de una primera aproximación a un tema tan complejo, como es el de la evaluación del desarrollo rural.

El concepto de desarrollo sostenible se ha incorporado en múltiples facetas de nuestra sociedad, en un intento de consolidación del crecimiento económico con la preservación del medio natural. En este sentido, es en el campo del desarrollo rural donde cobra especial importancia la aplicación de los principios de sostenibilidad, acompañándose de numerosas declaraciones y estudios, desde la década de los setenta. La sostenibilidad no supone una aportación novedosa en el campo de la planificación rural, pero sus principios se han cohesionado dentro de un concepto integrador que ha calado en las instituciones, agentes sociales y opinión pública, en un contexto en el que las políticas de desarrollo rural obligan a un equilibrio irrenunciable entre el crecimiento económico, la preservación ambiental y la equidad social.

Las políticas de desarrollo rural en las áreas LEADER (I y II) y PRODER (19911999) han tenido resultados y efectos desiguales, hecho que ha sido percibido pero no suficientemente evaluado. Es preciso conocer el grado de cumplimiento de los objetivos que se marcaron con la implantación de estas políticas y los efectos reales producidos en las distintas áreas, utilizando instrumentos que permitan compararlos entre ellos.

Las limitaciones de los indicadores tradicionales de desarrollo han sido criticadas desde los años setenta. La enorme relevancia de la Cumbre de Río 92 y el llamamiento de la Agenda 21 a crear indicadóres de sostenibilidad, han surtido efectos, al menos en el plano de la definición teórica. En el Plan de Aplicación de las Decisiones de la Cumbre Mundial sobre el Desarrollo Sostenible de Johannesburgo (2002), la aplicación de los indicadores confirma su importancia para medir los progresos hacia la sostenibilidad.

Por otro lado, el incremento en la dotación de fondos públicos ha traído aparejado una creciente demanda de técnicas de evaluación. En los años 70, comienzan a aplicarse de forma masiva a los proyectos de cooperación internacional (Banco Mundial, OCDE, etc.). En los años 80, se generalizan y perfeccionan las técnicas, y en los 90 , se adaptan a la cooperación española. En relación con los fondos comunitarios, la obligación de evaluación queda recogida en el Reglamento 4253/88 (artículos 25 y 26), donde se señala que la Comisión y los Estados miembros deben garantizar un seguimiento eficaz de la utilización de los fondos, así como una estimación y evaluación de las intervenciones. Igualmente, el Reglamento 2081/93 (artículos 26 y 31) establece que 
cada acción comunitaria deberá ser objeto de una apreciación previa, un seguimiento y una evaluación que permita valorar su repercusión respecto a los objetivos definidos para los Fondos Estructurales. Respecto a las intervenciones relacionadas con el desarrollo rural, es el Comité STAR el que adoptó en 1994 el documento "Orientaciones comunes para el seguimiento y las evaluaciones intermedias comunitarias".

Los programas de desarrollo rural, como muchas otras intervenciones de carácter estructural o financiados parcialmente con los Fondos Estructurales, están sujetos a una evaluación reglamentaria. La cual ha empezado a estar muy presente en el quehacer diario de los Grupos LEADER y en menor medida PRODER, y parece que la tendencia es que sea cada vez más habitual. LEADER I tuvo una evaluación al finalizar el Programa; LEADER II le añadió la evaluación intermedia; y para LEADER +, la Comisión ha incorporado a las anteriores, una tercera evaluación inicial. En pocos años se ha pasado de una a tres evaluaciones por programa, lo que evidencia la preocupación que la Comisión Europea tiene por evaluar este tipo de intervenciones (Esparcia, J., 2001).

La evaluación se ha convertido en un elemento estratégico de diferenciación, en la medida en que permite demostrar a sus propios beneficiarios, que la aplicación de los recursos públicos o privados, es la más adecuada posible.

Para evaluar es necesario un sistema de indicadores, que responden a cinco grandes tipos (Comisión Europea, 2005): relevancia (si se adecuan los objetivos del programa a las necesidades del área, sector o grupo de población), eficiencia (el esfuerzo invertido en términos de recursos físicos, humanos o financieros y los resultados obtenidos), eficacia (los impactos o resultados del programa respecto a sus objetivos), utilidad (los impactos del programa si han contribuido a las necesidades del área, sector o grupo de población) y la sostenibilidad (valorar la continuidad de los efectos después de la finalización del programa).

De todos ello, en este artículo, tan solo nos detendremos en los de eficacia (impacto de los proyectos-objetivos) y la eficiencia (inversión-resultado). Junto a los datos más objetivos y cuantitativos que emanan de este análisis, quisiéramos recalcar otra serie de aportaciones de carácter más subjetivo y que son fruto de la experiencia que el grupo de investigación adquirió durante el período 1998-2001. Desempeñar las funciones de "Antena Regional de la Unidad Española del Observatorio LEADER" al amparo del $\mathrm{MAPA}^{2}$ nos permitió un contacto directo con los GAL andaluces y un conocimiento de la realidad rural regional más allá de los contenidos estrictamente teóricos.

Todo ello en un futuro nos permitirá construir un índice de sostenibilidad rural, en el que se tengan en cuenta variables relacionadas con la actividad económica, la realidad social, el medio ambiente local y los recursos patrimoniales del territorio. 


\section{Metodología}

En el proyecto de investigación en el que se enmarca este artículo, el objetivo es la creación de un sistema de indicadores integrado en un proceso de planificación que permita un adecuado diagnóstico territorial y sectorial desde el punto de vista de la sostenibilidad, y que permita evaluar periódicamente la eficiencia de las medidas adoptadas, esto es, conocer el proceso de cambio cualitativo hacia el desarrollo sostenible. El sistema de indicadores debe observar una serie de criterios que garanticen su operatividad, contemplen el carácter integral y la perspectiva diacrónica del desarrollo sostenible, y midan la incidencia de las políticas de gestión públicas y privadas en el desarrollo sostenible. Con el objetivo de facilitar la creación del sistema de indicadores se seguirán tres pasos: uno, generación de posibles indicadores; dos, análisis y selección de los indicadores y tres, aplicación de los mismos.

En este articulo, nos centramos en la experiencia del desarrollo rural en el campo turístico (medida B3 de la iniciativa comunitaria LEADER II). Ésta ha sido la "estrella" de los programas de desarrollo rural, tanto por inversiones como por proyectos ejecutados. En Andalucía, al igual que en España, más del 50\% del total de los fondos en LEADER I se dirigieron al turismo. Posteriormente, en LEADER II los presupuestos se redistribuyeron más equitativamente entre todas las medidas en términos relativos (disminuyendo el turismo al 29\%), si bien en Andalucía se mantuvo por encima de la media nacional (31\%). Por ello, el turismo rural como estrategia de desarrollo rural no tiene en LEADER II la misma importancia que en la primera etapa, en la que se dedicaron más de la mitad de los recursos financieros a esta medida. En esta segunda fase se reduce a una tercera parte la inversión total en Andalucía. No obstante, se debe remarcar, que en términos absolutos, LEADER II dedicó 10.002 millones de pesetas, mientras que LEADER I, 7.402 millones de pesetas.

Los objetivos planteados en este articulo son:

- Detectar las comarcas más interesadas en desarrollar la oferta de turismo rural, por ello, de la inversión total generada en LEADER II se extrae el montante económico de la medida B3 y el volumen de proyectos ejecutados generados a raíz de dicha inversión.

- Comprobar el grado de eficiencia de la inversión en turismo rural en las comarcas andaluzas, respecto a los objetivos previstos en los respectivos Programas de Innovación Rural (PIR).

- Empezar a poner las bases de la generación de una estructura estadística que permita ir acumulando información relativa a diferentes indicadores (actividad económica, realidad social, medio ambiente local y recursos patrimoniales del territorio) para llegar a la construcción de un índice-resultado, que pueda ser un instrumento de análisis y diagnóstico. 
Se han seleccionado como indicadores, las variables o estimaciones que aporten una información agregada, sintética, verificable objetivamente y de tipo cuantitativo (número de proyectos e inversiones). Indicadores capaces de medir las realizaciones en términos de eficacia y eficiencia del programa LEADER II en las acciones turísticas ejecutadas.

En este caso se va a trabajar con la eficacia y la eficiencia como una primera aproximación hacia la creación de un índice-resultado, por lo tanto, los resultados que se obtengan en la generación de éste, deben ser entendidos como un paso inicial en el que sólo van a tenerse en cuenta ambas variables. Estos resultados en si ya son una base para establecer un primer nivel de resultados alcanzados por las comarcas, a los que indudablemente habrá que dotar de un mayor volumen de variables.

El método estadístico es un primer ensayo de aplicación en el que se pretende homogeneizar los datos a través del método de NADIR. Mientras que en el caso del indicador de eficacia (el éxito de los proyectos) lo más importante es que las comarcas hayan acometido el mayor número de proyectos $\left(\mathrm{v}_{1} \mathrm{y} \mathrm{v}_{2}\right)$, en el indicador de eficiencia $\left(\mathrm{v}_{3} \mathrm{y}_{4}\right)$ prima el menor coste generado posible para alcanzar una mayor eficiencia. Se determina un valor máximo y uno mínimo para cada una de las variables (basándose en los valores reales analizados).

En el caso del valor máximo (valor deseado), así como del mínimo (máxima privación) este método facilita la homogeneización de las diversas características, al reducir el conjunto de variables a un número a través del cual es posible establecer las correlaciones necesarias para la clasificación de las comarcas contempladas en el análisis. De esta forma, se facilita la categorización no sólo de un número de indicadores socioeconómicos sino también de las unidades espaciales.

$$
V_{\text {eficacia }}=\frac{\mathrm{N}-\text { Valor mínimo }}{\text { Valor máximo - Valor mínimo }} \quad \mathrm{V}_{\text {eficiencia }}=\frac{\text { Valor máximo }-\mathrm{N}}{\text { Valor máximo - Valor mínimo }}
$$

Esto nos permitirá ordenar las alternativas mediante el método del punto ideal con $\mathrm{P}_{2}$. Es un modelo que minimiza las diferencias que existen entre el nivel deseado y percibido para cada uno de los atributos que conforman las dimensiones de calidad del servicio. Se construyen los valores ideales de cada escenario y a $\mathrm{P}_{2}$ se le denomina de valoración ideal. Con ello se identifica cada $\mathrm{P}_{2}$ de las comarcas por la distancia entre el vector ideal y el siguiente vector. Cuanto menor sea la distancia entre ambos vectores, mayor será la posibilidad de alcanzar el óptimo de sostenibilidad.

$$
P_{2}=\sqrt{(1-v 1)^{2}+(1-v 2)^{2}+(1-v 3)^{2}+(1-v 4)^{2}}
$$


Asimismo el índice-resultado ( $\mathrm{Ir}$ ) es fruto del cociente $\mathrm{P}_{2}$ y el sumatorio de los valores de las comarcas LEADER. Consideramos índice-resultado como la integración de la eficacia y eficiencia. Con los datos obtenidos a partir de estos indicadores se diferencian cuatro categorías para las comarcas. De cara a la representación de cada uno de los valores alcanzados por las mismas, se han ido ordenando en la progresión de una recta de valores descendentes, desde el valor más alto que representa el nivel 4 del índice hasta el valor más bajo donde se encuentra el nivel 1.

La clasificación en los diferentes niveles se ha establecido comparando las comarcas entre si, y no con el valor medio regional. Por lo tanto, a la hora de realizar las agrupaciones se ha utilizado el valor de la centésima del resultado final, a fin de poder clasificar a los grupos en relación a su distancia hacia el óptimo, que sería el Ir=0.

\section{Indicadores de contexto}

En cuanto a la utilización de indicadores para el seguimiento y la evaluación de este tipo de programas, la Comisión Europea (COM (2001) 565 final) señala la necesidad de definir una batería de indicadores para la utilización de todos los países miembros. Entre los distintos tipos de indicadores se encuentran los indicadores de contexto, utilizados para examinar las políticas en un contexto económico y social más general.

Los indicadores de contexto se aplican a la totalidad de un territorio, población o categoría. Estos indicadores pueden situar el programa en perspectiva, mediante la medición de variables económicas, sociales o medioambientales apropiadas. Un indicador de contexto puede, por ejemplo, mostrar que un determinado programa ya no se justifica, pese a estar cumpliendo sus propios objetivos; $o$, en sentido inverso, que un determinado tipo de ayuda aún resulta procedente, aunque el indicador de programa señale un progreso limitado. La utilización de indicadores de contexto otros sólo deberán recabarse específicamente a efectos de la evaluación.

Retomando las variables seleccionadas para el estudio, eficacia y eficiencia representan un conjunto de pautas y normas para el análisis de las políticas de desarrollo rural. Son términos que se utilizan con significados polivalentes; sus definiciones generales incorporan conceptos ambiguos, de tal manera que se prestan a múltiples interpretaciones.

El Diccionario de la Lengua Española de la Real Academia señala que eficacia significa "virtud, actividad puede estar justificada de forma regular, mientras que, fuerza y poder para obrar... capacidad de lograr el efecto que se desea o espera". Moliner, $M$. 
(1998) interpreta esa definición y sugiere que dicho concepto "se aplica a las cosas o personas que pueden producir el efecto o prestar el servicio a que están destinadas".

Mokate, K. (2001) entiende que eficacia y efectividad son conceptos sinónimos y se pueden utilizar de forma intercambiable. Sin embargo, Cohen, E. y Franco, R. (1992) indican que la eficacia mide "el grado en que se alcanzan los objetivos $y$ metas... en la población beneficiaria, en un periodo determinado..." mientras que la efectividad constituye la relación entre los resultados (previstos y no previstos) y los objetivos. Así, estos autores proponen la efectividad como una medida que reconocería resultados diferentes a los que fueron esperados en la delimitación de los objetivos de la iniciativa.

En una línea similar se expresa la evaluación inicial de LEADER + que considera la eficacia como el "análisis de los efectos frente a los objetivos del programa evaluado" y la eficiencia como el "análisis de los efectos frente a los medios empleados (financieros o administrativos); esto es, desde el punto de vista económico, de qué modo los medios se ban convertido en realizaciones o resultados o ban generado un impacto" (Comisión Europea, 1999). De esta forma, una medida será eficaz si se alcanzan los objetivos, mientras que la eficiencia estará en relación en saber si se habrían logrado el mismo resultado con menos medios o más resultados con iguales medios.

Organismos internacionales como la IUCN (Unión Mundial para la Naturaleza) toman como eficacia a la relación de las "fortalezas de un grupo para cumplir con el propósito asumido. Tiene que ver con nuestra capacidad de transformación de la realidad. La eficacia nos indica en qué medida nos acercamos a la imagen objetivo o visión institucional". Mientras que la eficiencia es un concepto utilizado para referirse a la "relación existente entre el trabajo planificado y el ejecutado, que toma en cuenta el tiempo, la inversión económica, el personal asignado y los recursos materiales. Se refiere a nuestra capacidad de realizar el trabajo". Esto puede desembocar en proyectos que pueden tener un alto nivel de eficiencia (buenos productos con excelente manejo de los recursos), pero poco nivel de eficacia, o a la inversa tener capacidad de transformar la realidad (eficacia) pero sin utilizar adecuadamente sus recursos (ineficiencia). (IUCN, 2005).

Otros autores como Scriven, M. (1991) sugiere que si definimos la eficacia en términos del grado de cumplimiento de unos objetivos predefinidos, resulta ser un mero sinónimo de "éxito" y reconoce que se puede construir una medida que se refiera al logro de algún resultado que puede o no haber sido parte de los objetivos iniciales de la iniciativa. Por su parte, Reynaldo, A. (2002) manifiesta que "la eficiencia consiste en la medición de los esfuerzos que se requieren para alcanzar los objetivos. El costo, el tiempo, el uso adecuado de factores materiales y bumanos, cumplir con la calidad propuesta, constituyen elementos inberentes a la eficiencia". 
Aplicando todas estas definiciones a nuestro estudio, el indicador de eficacia de la iniciativa LEADER II en el sector turístico podría entenderse como el grado en que se consiguen los logros buscados a través del número de proyectos puestos en marcha; y por tanto, si se cumplen los objetivos de los PIR en función de las especificidades territoriales para los que fueron creados. Este indicador debería permitir determinar si la inversión realizada en esos proyectos ha sido óptima. Por otro lado, un rendimiento eficiente sería aquel que genera productos con menor insumo, o que manteniendo los niveles de inversión permita superar los resultados actuales a través de la creación de economías de escala.

Los indicadores de eficacia y eficiencia pueden contribuir a medir el impacto territorial que, sobre las comarcas rurales andaluzas, han tenido los proyectos e inversiones realizadas en materia de turismo rural durante la etapa LEADER II (tabla 1). La eficacia de esta iniciativa en el sector turístico podría entenderse como el grado en que se consiguen los logros buscados y por tanto, se cumplen los objetivos para los que fueron creados en relación a la superficie comarcal y poblacional.

Tabla 1. Indicadores de eficacia y eficiencia (medida B.3) en las comarcas LEADER II. 2001.

\begin{tabular}{|c|c|c|c|c|}
\hline \multirow{2}{*}{$\begin{array}{l}\text { GRUPOS DE DESARROLLO } \\
\text { RURAL DE ANDALUCIA } \\
\text { Comarcas }\end{array}$} & \multicolumn{2}{|c|}{$\begin{array}{l}\text { INDICADOR } \\
\text { DE EFICACIA }\end{array}$} & \multicolumn{2}{|c|}{ INDICADOR DE EFICIENCIA } \\
\hline & $\begin{array}{c}\text { Proy./ sup. } \\
\left(100 \mathrm{~km}^{2}\right)\end{array}$ & $\begin{array}{l}\text { Proy./ pob. } \\
(1.000 \text { hab })\end{array}$ & $\begin{array}{c}\text { Inv. }(€) / \\
\text { sup. }\left(\mathrm{km}^{2}\right)\end{array}$ & $\begin{array}{c}\text { Inv. }(€) / \\
\text { pob. }\end{array}$ \\
\hline Los Vélez & 1,31 & 1,19 & 599,74 & 54,64 \\
\hline Sierra Morena Cordobesa & 0,44 & 0,47 & 370,46 & 39,80 \\
\hline Los Pedroches & 0,39 & 0,23 & 268,85 & 16,29 \\
\hline Valle del Alto Guadiato & 1,84 & 0,79 & 782,27 & 33,46 \\
\hline Noreste de Granada & 0,76 & 0,41 & 772,35 & 41,47 \\
\hline Sierra de Aracena & 1,63 & 1,15 & 828,32 & 58,67 \\
\hline Andévalo Occidental & 0,19 & 0,12 & 319,60 & 19,65 \\
\hline Cuenca Minera Riotinto & 2,56 & 0,79 & $1.455,23$ & 44,83 \\
\hline Sierra Mágina & 2,03 & 0,61 & 820,04 & 24,77 \\
\hline El Condado de Jaén & 1,10 & 0,64 & 431,05 & 25,31 \\
\hline Sierra de las Nieves & 4,12 & 1,38 & $3.533,47$ & 118,16 \\
\hline Corredor de la Plata & 0,62 & 0,36 & 810,36 & 46,85 \\
\hline Sierra Morena de Sevilla & 0,25 & 0,20 & 201,08 & 15,79 \\
\hline Las Alpujarras & 1,04 & 0,41 & 584,19 & 23,09 \\
\hline Sierra de Cádiz & 2,20 & 0,37 & $1.372,08$ & 23,08 \\
\hline Los Alcornocales & 0,53 & 0,14 & 796,61 & 21,54 \\
\hline Guadix & 1,03 & 0,40 & $1.059,88$ & 40,83 \\
\hline Poniente Granadino & 0,54 & 0,14 & 482,25 & 12,14 \\
\hline Sierra de Segura & 3,37 & 2,16 & $1.169,40$ & 74,73 \\
\hline La Axarquía & 6,57 & 1,08 & $5.268,60$ & 86,53 \\
\hline Serranía de Ronda & 2,63 & 0,61 & $3.147,14$ & 73,49 \\
\hline Estepa- Sierra Sur & 0,34 & 0,06 & 220,08 & 3,96 \\
\hline
\end{tabular}

Fuente: Elaboración propia. 
Respecto al indicador de eficacia, el número de proyectos medios ejecutados por superficie en Andalucía de las 517 acciones analizadas es de 1,20 proyectos por cada $100 \mathrm{~km}^{2}$. Las comarcas más eficaces teniendo en cuenta la calidad y oportunidad brindada para el territorio son La Axarquía $\left(6,57\right.$ proyectos/ $\left.100 \mathrm{~km}^{2}\right)$, Sierra de las Nieves $(4,12)$, Sierra de Segura $(3,37)$ y Cuenca Minera $(2,56)$. Mayoritariamente son comarcas con una superficie relativamente pequeña, en torno a $650 \mathrm{~km}^{2}$, pero que han llevado a cabo mayor número de proyectos. En cambio, en las de mayor extensión, Sierra Morena de Sevilla $(0,25)$, Estepa-Sierra Sur $(0,34)$, Los Pedroches $(0,39)$ y Sierra Morena cordobesa $(0,44)$, se han realizado menos proyectos.

Asimismo, si dentro de este indicador consideramos no sólo la eficacia respecto a la superficie, sino también a la población, se obtiene una media de 0,48 proyectos por cada 1.000 habitantes. En este caso, la mayor eficacia la alcanzan Sierra de Segura $(2,16$ proyectos $/ 1.000$ hab.), Sierra de las Nieves $(1,38)$, Los Vélez $(1,19)$, Sierra de Aracena $(1,15)$. El menor impacto corresponde a las comarcas de Estepa- Sierra Sur $(0,06)$, Andévalo occidental $(0,12)$, Poniente Granadino y Los Alcornocales $(0,14$ respectivamente), teniendo en cuenta este indicador.

La eficiencia considerada por algunos autores como la relación existente entre el trabajo desarrollado, el tiempo invertido, la inversión realizada en hacer algo y el resultado logrado, nos permite decir que en este caso lo que se valora es la productividad. Para ello se ha considerado que la media andaluza ha sido de 841 euros de inversión turística por cada $100 \mathrm{~km}^{2}$. Con relación a ello, una serie de comarcas han visto incrementada su productividad, nos referimos a La Axarquía (5.269), Sierra de las Nieves (3.533), Serranía de Ronda (3.147), y en menor medida, Cuenca Minera (1.455), Sierra de Cádiz (1.372), Sierra de Segura (1.169), o comarca de Guadix (1.060). De estas siete comarcas, las cinco primeras, se beneficiaron de la iniciativa LEADER I, y ello les ha permitido encontrarse en una posición de privilegio respecto al resto, pues con el anterior programa crearon las bases de las infraestructuras turísticas, que frecuentemente son los proyectos más costosos, y por tanto, han hecho que sean más eficientes. En esta segunda etapa se han especializado en la diversificación de actividades complementarias y en la mejora de la calidad turística.

Dentro de este indicador, al igual que se consideraba con el de eficacia, se ha seleccionado la inversión turística respecto a la población. Y los resultados medios andaluces son de 33,7 euros de inversión por cada habitante, situándose el valor máximo en la Sierra de las Nieves en 118 euros/habitante, y el mínimo en 4 euros/habitante en la comarca de Estepa-Sierra Sur. Por tanto, los resultados más eficientes se alcanzan cuando se hace un uso adecuado de las inversiones en relación con superficie y población.

Ambos índices, eficacia y eficiencia, deben ser abordados conjuntamente para comenzar a construir un indice-resultado. 


\section{Primera aproximación en la construcción de un índice-resultado en materia de turismo}

El índice resultado es la fusión de la información contenida en varias variables (eficacia y eficienca) en una sola expresión numérica. Es adimensional pues resulta de la adición ponderada, según el procedimiento que se elija, de diversas unidades de medida. Un índice posee las mismas características que un indicador, pero el beneficio que produce es aún mayor puesto que se obtiene una síntesis de la información relevante sobre el turismo rural.

Teniendo en cuenta el método estadístico de NADIR para la homogeneización de variables, y considerando el indicador de eficacia $\left(v_{1} y_{2}\right)$ y el indicador de eficiencia $\left(\mathrm{v}_{3} \mathrm{y} \mathrm{v}_{4}\right)$ se establece una categorización de las comarcas andaluzas mediante el método del punto ideal $\left(\mathrm{P}_{2}\right)$. Con todo ello llegamos al índice resultado (Ir), considerando que el óptimo es Ir $=0$.

Tabla 2. Índice-resultado en materia de turismo.

\begin{tabular}{|c|c|c|c|c|c|c|}
\hline GRUPOS & $\mathrm{V}_{1}{ }^{3}$ & $V_{2}^{4}$ & $\mathrm{~V}_{3}^{5}$ & $\mathbf{V}_{4}^{6}$ & $\mathbf{P}_{2}$ & Ir \\
\hline Los Vélez & 0,18 & 0,54 & 0,92 & 0,56 & 1,0470 & 0,0391 \\
\hline Sierra Morena Cordobesa & 0,04 & 0,20 & 0,97 & 0,69 & 1,2915 & 0,0482 \\
\hline Los Pedroches & 0,03 & 0,08 & 0,99 & 0,89 & 1,3385 & 0,0500 \\
\hline Valle del Alto Guadiato & 0,26 & 0,35 & 0,89 & 0,74 & 1,0291 & 0,0384 \\
\hline Noreste de Granada & 0,09 & 0,17 & 0,89 & 0,67 & 1,2825 & 0,0479 \\
\hline Sierra de Aracena & 0,23 & 0,52 & 0,88 & 0,52 & 1,0366 & 0,0387 \\
\hline Andévalo Occidental & 0,00 & 0,03 & 0,98 & 0,86 & 1,4026 & 0,0524 \\
\hline Cuenca Minera Riotinto & 0,37 & 0,35 & 0,75 & 0,64 & 1,0062 & 0,0376 \\
\hline Sierra Mágina & 0,29 & 0,26 & 0,88 & 0,82 & 1,0472 & 0,0391 \\
\hline El Condado de Jaén & 0,14 & 0,28 & 0,95 & 0,81 & 1,1372 & 0,0425 \\
\hline Sierra de las Nieves & 0,62 & 0,63 & 0,34 & 0,00 & 1,3112 & 0,0490 \\
\hline Corredor de la Plata & 0,07 & 0,14 & 0,88 & 0,62 & 1,3282 & 0,0496 \\
\hline Sierra Morena de Sevilla & 0,01 & 0,07 & 1,00 & 0,90 & 1,3655 & 0,0510 \\
\hline Las Alpujarras & 0,13 & 0,17 & 0,92 & 0,83 & 1,2163 & 0,0454 \\
\hline Sierra de Cádiz & 0,32 & 0,15 & 0,77 & 0,83 & 1,1296 & 0,0422 \\
\hline Los Alcornocales & 0,05 & 0,04 & 0,88 & 0,85 & 1,3627 & 0,0509 \\
\hline Guadix & 0,13 & 0,16 & 0,83 & 0,68 & 1,2612 & 0,0471 \\
\hline Poniente Granadino & 0,05 & 0,04 & 0,94 & 0,93 & 1,3533 & 0,0505 \\
\hline Sierra de Segura & 0,50 & 1,00 & 0,81 & 0,38 & 0,8195 & 0,0306 \\
\hline La Axarquía & 1,00 & 0,49 & 0,00 & 0,28 & 1,3370 & 0,0499 \\
\hline Serranía de Ronda & 0,38 & 0,26 & 0,42 & 0,39 & 1,2770 & 0,0477 \\
\hline Estepa- Sierra Sur & 0,02 & 0,00 & 1,00 & 1,00 & 1,3978 & 0,0522 \\
\hline
\end{tabular}


Un paso más en la metodología de los indicadores es llegar a establecer el perfil (gráfico 1), que consiste en la selección de varios indicadores, en su conjunto, o por partes, que sirvan para dar cuenta del estado de la cuestión para un sistema, en este caso, del turismo en el desarrollo rural. De tal manera podríamos aproximarnos a la creación de un índice-resultado fruto de la combinación de los indicadores aportados (eficacia y eficiencia). Dicho índice será más completo cuanto mayor sea el número de variables utilizadas en su construcción.

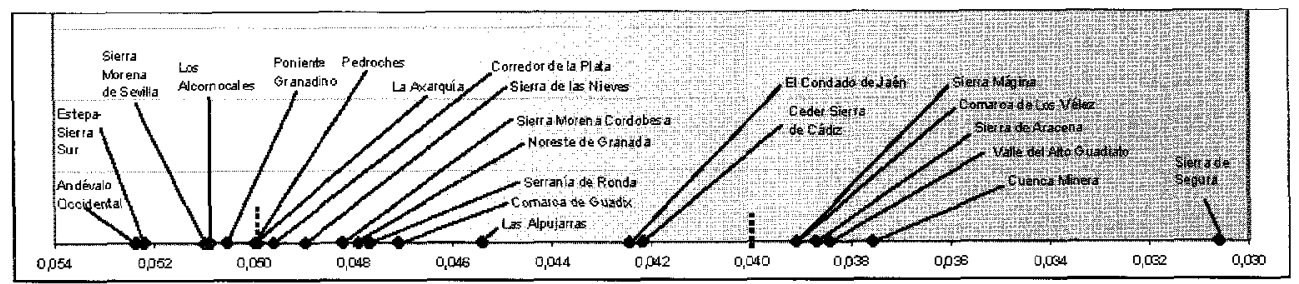

Grafico 1. Representación del perfil del Ir en Andalucía.

Fuente: Elaboración propia.

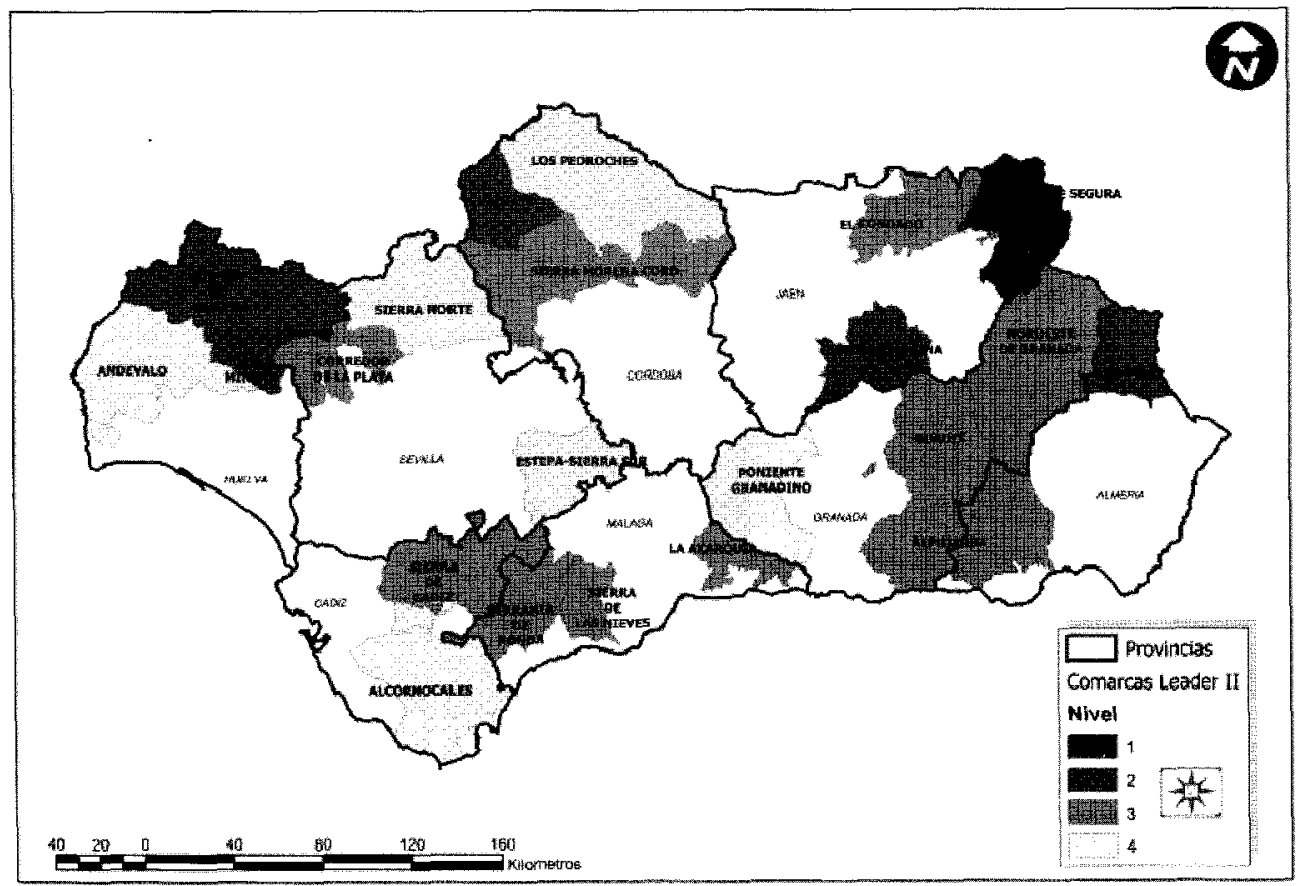

Mapa 1. Distribución por niveles de las comarcas LEADER II de Andalucía.

Fuente: Elaboración propia. 
Tal y como podemos observar en el mapa 1, aparecen las comarcas agrupadas en categorías o niveles en función de los resultados obtenidos, según la proporción de mayor número de proyectos y superior inversión ejecutada en turismo. En líneas generales, podemos decir que las comarcas que gozan de mayor nivel, no son las consideradas destinos turísticos rurales consolidados, ni tienen que ser las que se ubican en espacios naturales protegidos, sino que la prioridad de las convocatorias de los CEDERs, la inquietud de los empresarios y el propio territorio han propiciado esta situación.

\section{Nivel 1. Comarcas con índice-resultado alto $(\mid \mathrm{r}<0,0310)$}

La comarca que alcanza el resultado más elevado es La Sierra de Segura, ya que es la que más proyectos ha ejecutado ( 65 actuaciones turísticas) con una menor inversión (2.253.442 euros), es decir, con un reparto más equitativo proyectos/inversión. Los objetivos planteados en el PIR eran: rehabilitar y poner en valor el patrimonio arquitectónico, actuar como motor de la economía de la comarca, y crear una imagen de marca de turismo rural, basada en el contacto con la vida rural, la explotación agrícola y la naturaleza; y de su cumplimiento se deriva el índice 0,0306 .

En esta comarca existían con anterioridad bases estratégicas del sector turístico, por tener experiencia en otros programas de actuación (Programa de Desarrollo Integral de Turismo Rural -PRODITUR- desarrollado por la Junta de Andalucía, instrumento de financiación del Instituto de Fomento de Andalucía -IFA-, etc.). A ello hay que añadirle su favorable emplazamiento geográfico en el Parque Natural de Sierra de Cazorla, Segura y Las Villas.

Los proyectos más numerosos se han centrado en incrementar la oferta de acogida (hoteles, casas rurales, apartamentos y camping), así como la práctica de todo tipo de formas de turismo al aire libre (parapente, ala delta, actividades náuticas, rutas en todoterreno) para diversificar la oferta complementaria.

\section{Nivel 2. Comarcas con índice-resultado medio-alto $(\mid r<0,04)$}

En el segundo nivel se encuentran una serie de comarcas atipicas desde el punto de vista turístico, pero que han aprovechado bien los recursos disponibles a través de esta iniciativa comunitaria. Nos referimos a Cuenca Minera $(0,0376)$, Alto Guadiato $(0,0384)$, Sierra de Aracena $(0,0387)$, Los Vélez y Sierra Mágina (ambos 0,0391). Comarcas, unas con tradición minera y otras, protegidas con la figura de Parque Natural, todos han sabido diversificar su oferta con nuevos productos turísticos industriales y naturales. 


\section{Nivel 3. Comarcas con índice-resultado medio $($ Ir $<0,05)$}

Las comarcas de nivel 3 satisfacen los objetivos turísticos que planteaban en sus PIR, aunque en muchos casos, sin medir los recursos para alcanzarlos. Ello supone una baja rentabilidad de sus inversiones.

Dentro de este nivel demos establecer una segunda clasificación:

a) En líneas generales, las comarcas rurales que se beneficiaron de la iniciativa LEADER I se encuentran en una posición intermedia, pues aquel programa crearon las bases de las infraestructuras turísticas, es decir, los proyectos más costosos. En esta segunda etapa se han especializado en la diversificación de actividades complementarias y/o mejorar la calidad turística. En este caso se encuentran la Sierra de Cádiz $(0,0422)$, Alpujarras $(0,0454)$, Serranía de Ronda $(0,0477)$ y La Axarquía $(0,0499)$.

b) En cambio, las comarcas que son por primera vez subvencionadas, han tenido que invertir mayor presupuesto en infraestructuras turísticas básicas, ante la inexistencia de las mismas o escasez para ofrecer al visitante un buen producto. En este segundo grupo del nivel 3 se encuentran El Condado de Jaén $(0,0425)$, Comarca de Guadix $(0,0471)$, Noreste de Granada $(0,0479)$, Sierra Morena cordobesa $(0,0482)$, Sierra de las Nieves $(0,0490)$ y Corredor de la Plata $(0,0496)$.

Nivel 4. Comarcas con indice-resultado bajo (Ir $>0,05)$

Son comarcas con una limitada posibilidad de competir en materia turística, debido a que su especialización productiva se ha centrado tradicionalmente en otros sectores económicos. Estas comarcas son Los Pedroches $(0,05)$, Poniente granadino $(0,0505)$, Los Alcornocales (0,0509), Sierra Morena de Sevilla $(0,0510)$, Estepa- Sierra Sur $(0,0522)$ y Andévalo occidental $(0,0524)$. Han realizado un escaso número de proyectos con un presupuesto bajo, ya que de todas las medidas de actuación contempladas en LEADER II, el turismo ha sido la menos relevante para todos ellos.

\section{Síntesis}

En toda España podríamos hablar del turismo rural como una actividad económica de éxito y rápida implantación territorial en la última década. Su importancia como instrumento de diversificación de las economías rurales y como nueva fuente de ingresos y empleos es por todos reconocida. En el caso de Andalucía, esta actividad 
ha conseguido generar unos flujos de inversión y de personas hasta hace poco desconocidos. En unos territorios rurales, donde la ausencia de iniciativa privada y la falta de tejido y cultura empresarial habian sido las notas predominantes, la nueva modalidad turística es abrazada con desigual fuerza por las comarcas.

No ha sido el objetivo de este trabajo señalar los logros alcanzados en términos económicos, sociales o ambientales, de la mano del turismo rural en nuestras comarcas. Sino conocer el grado de utilidad que un programa de desarrollo rural como LEADER II ha tenido para dichos espacios a través de una de sus medidas de actuación. En este caso, exclusivamente de la medida B.3 (turismo rural). Paralelamente se ha tratado de conocer cual ha sido el uso que de este programa, entendido como herramienta de financiación, se ha hecho desde cada Grupo de Acción Local (GAL), así como las razones que han justificado el mismo.

No hemos pretendido poner sobre la mesa los destinos turísticos rurales más demandados o con una más amplia y diversificada oferta de la región, que por otro lado son sobradamente conocidos. La finalidad era establecer categorías entre las comarcas andaluzas acogidas a LEADER II en función de su grado de eficacia y de eficiencia en materia de turismo, teniendo tan solo en cuenta el número de proyectos ejecutados y la inversión requerida para ello.

El haber seleccionado como indicadores de contexto, la eficacia (el grado en que se han conseguido los logros buscados a través del mayor número de proyectos puestos en marcha) y la eficiencia (la óptima inversión realizada en esos proyectos) en materia turística nos ha permitido establecer un índice-resultado.

Siempre teniendo en cuenta que la información aquí aportada puede completarse aun más si añadimos otras variables que doten de mayor significado al índice. Dicho índice nos muestra dos hechos de interés:

1. Las comarcas que gozan de mayor nivel, no son las más conocidas como destinos turísticos rurales, consolidados ni tienen porque ser las ubicadas en espacios naturales protegidos, sino que han sido otros factores los que la posicionan en dichos niveles: la prioridad de las convocatorias de los CEDERs, la inquietud de los empresarios y el propio territorio han propiciado esta situación.

2. Las comarcas, que por primera vez son subvencionadas en LEADER II, suelen invertir en infraestructuras turísticas y servicios de alojamiento, siendo su inversión más costosa ante la inexistencia de las mismas o escasez para ofrecer al visitante un buen producto. Mientras, que aquellas que ya tienen las bases turísticas (LEADER I), se especializan en la segunda convocatoria, en la diversificación de actividades complementarias y/o mejorar la calidad turística. 
En consecuencia los indicadores y los índices son válidos para analizar el impacto producido por las diferentes políticas aplicadas en el mundo rural, pero para continuar el camino hacia un desarrollo sostenible hay que contar con otros factores que lo impulsen como el capital social y cultural, que aporten una visión integrada del territorio.

\section{Notas}

1 Este artículo se enmarca en el Proyecto "Construcción de una Red de Indicadores de Sostenibilidad para la evaluación dinámica de los procesos de desarrollo mural áreas LEADER y PRODER" con referencia AGL200304540, cofinanciado por el Ministerio de Educación y Ciencia (MEC) y la Comisión Europea (FEDER).

2 Ministerio de Agricultura, Pesca y Alimentación. Unidad Española del Observatorio Europeo LEADER en España. TRAGSATEC. "Antena Regional de la Unidad Española del Observatorio LEADER", a través del proyecto $11 / 45\left(n^{\circ} 126 / 98,55 / 99,15 / 00\right.$ y 49/01)

$3 \mathrm{~V}_{\mathrm{I}}=$ vector de proyectos/superficie.

$4 \mathrm{~V}_{2}=$ vector de proyectos/población.

$5 \mathrm{~V}_{3}=$ vector de inversión/superficie.

$6 \mathrm{~V}_{4}=$ vector de inversión/población.

\section{Bibliografía}

Buitrago, E y Foronda, C. (2000): Análisis de resultados de las políticas de desarrollo rural. Revista de Estudios Andaluces $n^{\circ} 24$. Universidad de Sevilla, p. 137-172.

Cohen, E. y Franco, R. (1992): Evaluación de proyectos sociales. México. Siglo Veiuntiuno.

Comisión Europea (1997): La evaluación global de un proyecto LEADER: Western Isles, Skye and Lochalsh. (Escocia, Reino Unido). Rural Europe. AEIDL

Comisión Europea (1999): Evaluación de los programas de desarrollo miral 2000-2006 financiados con cargo al Fondo Europeo de Orientación y Garantía Agrícola. Directrices. Dirección General de Agricultura.

Comisión Europea (2000): "Cuestionario para evaluar el Planteamiento LEADER" Seminario "Mejorar la calidad de las evaluaciones expost de LEADER II". 23-24 de noviembre de 2001. Bruselas.

Comisión Europea (2000): Indicadores de Seguimiento y Evaluación: Orientaciones metodológicas. El nuevo periodo de programación 2000-2006: Documentos de trabajo metodológicos. Dirección General XVI. Política Regional y Cohesión. 
Comisión Europea (2000): Proyecto de evaluación a posteriori de la Iniciativa Comunitaria LEADER II. Directrices para la evaluación.

Comisión Europea (2005): Orientaciones para la evaluación previa de los programas. (consulta:marzo) http://europa.eu.int/comm/agriculture/rur/LEADERplus/guidelines/

Esparcia, J. (2001): La evaluación de programas de desarrollo rural, en Pillet, F. y Plaza, J. (coord.) Lecciones de desarrollo rural. Universidad de Castilla la Mancha, p. 159-167.

Gużmán, M., Cobacho, M.A. y Martínez, E. (2001): Metodología para la evaluación de programas de desarrollo rural. Revista electrónica infoagro (fecha de consulta: junio 2002) (www.infoagro.com/)

IUCN (2005): Hacia la equidad. Unión mundial para la naturaleza. (fecha de consulta: marzo 2005) (http://www.generoyambiente.org/ES/publicaciones_uicn/eq uidadesp/m04/i.pdf)

Kearney, B. (2000): "Evaluación ex post del programa LEADER II en Irlanda. Resumen de métodos y resultados". Observatorio Europeo LEADER. Seminario "Mejorar la calidad de las evaluaciones ex - post de LEADER II".

Márquez, D., Foronda, C. y García, A. (1999): Las zonas agrarias intermedias ante el des- afio de la Agenda 2000, en Ramos Leal (coord.) El desarrollo rural en la Agenda 2000. Cap. 18. Ministerio de Agricultura, Pesca y Alimentación. Secretaria General Técnica, p. 393-406.

Ministerio de Medio Ambiente (2000): Indicadores ambientales. Una propuesta para España. Secretaria General Técnica. Madrid.

Mokate, K.M. (2001): Eficacia, eficiencia, equidad y sostenibilidad: ¿qué queremos decir? Serie Documentos de Trabajo del Instituto Interamericano para el Desarrollo Social INDES I-24. Washington (fecha de consulta: marzo 2002) (http://indes.iadb.org/pub/t24.PDF).

Moliner, M. (1998): Diccionario del uso del español. Editorial Gredos, S.A.

Reynaldo, A. (2002): Eficacia y eficiencia. (fecha de consulta: marzo 2003) (http://winred.com/EP/ articulos/menagement/a 1409.html)

Scriven, M. (1991): Evaluation Thesaurus. Fourth Edition. Newbury Park: Sage Publications.

Thirion, S. (2000): "El método SAP en Portugal (Sistematización de la Autoevaluación Participativa" Observatorio Europeo LEADER. Seminario "Mejorar la calidad de las evaluaciones ex - post de LEADER II". 